\title{
K3 SURFACES WITH PICARD NUMBER 2, SALEM POLYNOMIALS AND PELL EQUATION
}

\author{
KENJI HASHIMOTO, JONGHAE KEUM AND KWANGWOO LEE
}

\begin{abstract}
If an automorphism of a projective K3 surface with Picard number 2 is of infinite order, then the automorphism corresponds to a solution of Pell equation. In this paper, by solving this equation, we determine all Salem polynomials of symplectic and anti-symplectic automorphisms of projective K3 surfaces with Picard number 2.
\end{abstract}

\section{INTRODUCTION}

A compact complex surface $X$ is called a $\mathrm{K} 3$ surface if it is simply connected and has a nowhere vanishing holomorphic 2 -from $\omega_{X}$. By the global Torelli theorem the automorphism group of a K3 surface is determined, up to subquotient of finite index, by its Picard lattice. Suppose that a K3 surface $X$ is projective and has Picard number 2. Galluzzi, Lombardo and Peters GLP applied the classical theory of binary quadratic forms (cf. [D, J]) to prove that the automorphism group $\operatorname{Aut}(X)$ is finite cyclic, infinite cyclic or infinite dihedral.

Suppose that $\varphi \in \operatorname{Aut}(X)$ has infinite order and is either symplectic or antisymplectic, where we have $\varphi^{*} \omega_{X}=\varepsilon \omega_{X}$ and $\varphi$ is said to be symplectic (resp. antisymplectic) if $\varepsilon=1$ (resp. $\varepsilon=-1$ ). In the present paper, we determine all Salem polynomials of such automorphisms. The Salem polynomial of $\varphi$ is defined as the characteristic polynomial of $\left.\varphi^{*}\right|_{S_{X}}$ in this case. Here $S_{X}$ denotes the Picard lattice of $X$. Since $\operatorname{det}\left(\left.\varphi^{*}\right|_{S_{X}}\right)=1$, it is enough to determine $\operatorname{trace}\left(\left.\varphi^{*}\right|_{S_{X}}\right)$, and the Salem polynomial of $\varphi$ is given as

$$
x^{2}-t x+1, \quad t=\operatorname{trace}\left(\left.\varphi^{*}\right|_{S_{X}}\right) .
$$

We construct an automorphism $\varphi$ whose trace on $S_{X}$ is as in the condition (2) in Main Theorem below. We use a result on class numbers of real quadratic fields for the construction.

We apply the theory of binary quadratic forms to study actions on $S_{X}$ (cf. [GLP]). The (induced) action $\left.\varphi^{*}\right|_{S_{X}}$ of $\varphi$ on $S_{X}$ as above corresponds to an integer solution $(u, v)$ to the Pell equation

$$
u^{2}-D v^{2}=4, \quad D:=-\operatorname{disc}\left(S_{X}\right)>0 .
$$

More precisely, let

$$
\left(\begin{array}{cc}
2 a & b \\
b & 2 c
\end{array}\right), \quad a, b, c \in \mathbb{Z}
$$


be the intersection matrix of $S_{X}$ (under some basis). Then $D=b^{2}-4 a c$ and $\left.\varphi^{*}\right|_{S_{X}}$ is represented as the matrix

$$
g_{u, v}=\left(\begin{array}{cc}
(u-b v) / 2 & -c v \\
a v & (u+b v) / 2
\end{array}\right),
$$

where $(u, v)$ is an integer solution to the Pell equation (1.2) with $u>0$ (see Proposition 2.2). Moreover, we show

$$
(u, v)=\left(\alpha^{2}-2 \varepsilon, \alpha \beta\right), \quad \alpha^{2}-D \beta^{2}=4 \varepsilon
$$

for some integers $\alpha, \beta$ (see Proposition 4.1). In particular, we have $g_{u, v}=g_{\alpha, \beta}^{2}$. Our goal is to determine all possible values of

$$
\operatorname{trace}\left(\left.\varphi^{*}\right|_{S_{X}}\right)=u=\alpha^{2}-2 \varepsilon
$$

The result is stated as follows:

Main Theorem. For $\varepsilon \in\{ \pm 1\}$ and $u \in \mathbb{Z}$, the following conditions are equivalent:

(1) $u=\operatorname{trace}\left(\left.\varphi^{*}\right|_{S_{X}}\right)$ for some automorphism $\varphi$ of a projective K3 surface $X$ with Picard number 2 such that $\operatorname{ord}(\varphi)=\infty$ and $\varphi^{*} \omega_{X}=\varepsilon \omega_{X}$.

(2) $u=\alpha^{2}-2 \varepsilon$ for some $\alpha \in A_{\varepsilon}$, where

$$
A_{\varepsilon}= \begin{cases}\mathbb{Z}_{\geq 4} & \text { if } \varepsilon=1, \\ \mathbb{Z}_{\geq 4} \backslash\{5,7,13,17\} & \text { if } \varepsilon=-1 .\end{cases}
$$

Remark 1.1. (i) For a given trace $u$ as in Main Theorem, we have only finitely many pairs $(X, \varphi)$ with $\operatorname{trace}\left(\left.\varphi^{*}\right|_{S_{X}}\right)=u$, up to equivariant deformation. Indeed, for a given $u$ there are only finitely many $D$ 's satisfying the Pell equation (1.2) for some integer $v$, and for a given $D$ there are only finitely many lattices of rank 2 with discriminant $-D$ up to isomorphism (see [CS, Chap. 15]). Each of such lattices corresponds to a connected moduli space (of dimension $20-\operatorname{rank} S_{X}=18$ ) of $(X, \varphi)$ such that $S_{X}$ is isomorphic to the given lattice. (ii) An automorphism of infinite order of a projective K3 surface $X$ with Picard number 2 always acts on the Picard lattice $S_{X}$ with determinant 1 .

As an application, we show that every projective K3 surface with Picard number 2 admitting a fixed-point-free automorphism must have the same Picard lattice as the K3 surfaces with the Cayley-Oguiso automorphism [O, FGvGvL], and that the action of the automorphism on the Picard lattice is the same as that of the Cayley-Oguiso automorphism (see Section 5).

The structure of this paper is as follows: We recall some results on lattices and their isometries in Section 2, and K3 surfaces and their automorphisms in Section 3 . We prove Main Theorem in Section 4, Finally, in Section 5, a few applications of our result are discussed.

Acknowledgment. This work was supported by National Research Foundation of Korea (NRF-2007-0093858). The first author is partially supported by JSPS Grantin-Aid for Young Scientists (B), No. 17K14156. We would like to thank Professor Shigeru Mukai for useful discussion. 


\section{LATTICES}

A lattice is a free $\mathbb{Z}$-module $L$ of finite rank equipped with a symmetric bilinear form $\langle\rangle:, L \times L \rightarrow \mathbb{Z}$. If $x^{2}:=\langle x, x\rangle \in 2 \mathbb{Z}$ for any $x \in L$, a lattice $L$ is said to be even. We fix a $\mathbb{Z}$-basis of $L$ and identify the lattice $L$ with its Gram matrix $Q_{L}$ under this basis. The discriminant $\operatorname{disc}(L)$ of $L$ is $\operatorname{defined}$ as $\operatorname{det}\left(Q_{L}\right)$, which is independent of the choice of basis. A lattice $L$ is called non-degenerate if $\operatorname{disc}(L) \neq 0$ and unimodular if $\operatorname{disc}(L)= \pm 1$. For a non-degenerate lattice $L$, the signature of $L$ is defined as $\left(s_{+}, s_{-}\right)$, where $s_{+}$(resp. $s_{-}$) denotes the number of the positive (resp. negative) eigenvalues of $Q_{L}$. An isometry of $L$ is an isomorphism of the $\mathbb{Z}$-module $L$ preserving the bilinear form. The orthogonal group $O(L)$ of $L$ consists of the isometries of $L$. We consider $L$ as $\mathbb{Z}^{n}$ (column vectors) with Gram matrix $Q_{L}$ and use the following identification:

$$
O(L)=\left\{g \in G L_{n}(\mathbb{Z}) \mid g^{T} \cdot Q_{L} \cdot g=Q_{L}\right\}, \quad n=\operatorname{rank} L .
$$

For a non-degenerate lattice $L$, the discriminant group $A(L)$ of $L$ is defined by

$$
A(L):=L^{*} / L, \quad L^{*}:=\{x \in L \otimes \mathbb{Q} \mid\langle x, y\rangle \in \mathbb{Z}(\forall y \in L)\} .
$$

Let $K$ be a sublattice of a lattice $L$, that is, $K$ is a $\mathbb{Z}$-submodule of $L$ equipped with the restriction of the bilinear form of $L$ to $K$. If $L / K$ is torsion-free as a $\mathbb{Z}$-module, $K$ is said to be primitive. For a non-degenerate lattice $L$ of signature $(1, k)$ with $k \geq 0$, we have the decomposition

$$
\left\{x \in L \otimes \mathbb{R} \mid x^{2}>0\right\}=C_{L} \sqcup\left(-C_{L}\right)
$$

into two disjoint cones. Here $C_{L}$ and $-C_{L}$ are connected components. We define

$$
O^{+}(L):=\left\{g \in O(L) \mid g\left(C_{L}\right)=C_{L}\right\}, \quad S O^{+}(L):=O^{+}(L) \cap S O(L),
$$

where $S O(L)$ is the subgroup of $O(L)$ consisting of isometries of determinant 1 . The group $O^{+}(L)$ is a subgroup of $O(L)$ of index 2 .

Lemma 2.1. Let $L$ be a non-degenerate even lattice of rank $n$. For $g \in O(L)$ and $\varepsilon \in\{ \pm 1\}, g$ acts on $A(L)$ as $\varepsilon \cdot$ id if and only if $\left(g-\varepsilon \cdot I_{n}\right) \cdot Q_{L}^{-1}$ is an integer matrix.

Proof. As in (2.1), we consider $L$ as $\mathbb{Z}^{n}$ with Gram matrix $Q_{L}$. Then $L^{*}$ is generated by the columns of $Q_{L}^{-1}$. This implies the assertion.

In the rest of this section, we recall a few results related to lattices of signature $(1,1)$, or indefinite binary quadratic forms.

Proposition 2.2. ([D, J]) Let $L$ be an even lattice of signature $(1,1)$ :

$$
L:=\left(\begin{array}{cc}
2 a & b \\
b & 2 c
\end{array}\right), \quad D:=-\operatorname{disc}(L)=b^{2}-4 a c>0 .
$$

Then $\mathrm{SO}^{+}(L)$ consists of the elements of the form

$$
g=\left(\begin{array}{cc}
(u-b v) / 2 & -c v \\
a v & (u+b v) / 2
\end{array}\right) .
$$

Here $(u, v)$ is any solution of the positive Pell equation

$$
u^{2}-D v^{2}=4
$$

with $u, k v \in \mathbb{Z}$ and $u>0$, where $k:=\operatorname{gcd}(a, b, c)$. If $D$ is a square number (resp. not a square number), then $\mathrm{SO}^{+}(L)$ is isomorphic to a trivial group (resp. $\mathbb{Z}$ ). 
Remark 2.3. In Proposition 2.2, $D$ is a square number if and only if there exists $v \in L$ with $v^{2}=0$.

Remark 2.4. Pell equation is usually considered as a Diophantine equation, that is, only integer solutions are admitted. However, in Proposition 2.2, we need non-integer solutions to find all elements in $S O^{+}(L)$. Of course we have usual Pell equation by replacing $a, b, c$ and $D$ by $a / k, b / k, c / k$ and $D / k^{2}$.

Remark 2.5. In Proposition [2.2, the eigenvalues of $g$ are $(u \pm v \sqrt{D}) / 2$ and the solution $\left(u^{\prime}, v^{\prime}\right)$ of (2.7) corresponding to $g^{k}(k \in \mathbb{Z})$ satisfies

$$
\frac{u^{\prime}+v^{\prime} \sqrt{D}}{2}=\left(\frac{u+v \sqrt{D}}{2}\right)^{k} \text {. }
$$

Let $\left(u_{0}, v_{0}\right)$ be the smallest positive solution of (2.7), that is, $\left(u_{0}, v_{0}\right)$ is the solution with $u_{0}, v_{0}>0$ which minimizes $u_{0}$ (or $v_{0}$ ). Then $S O^{+}(L)$ is generated by $g_{0}$ corresponding to $\left(u_{0}, v_{0}\right)$.

We define

$$
\begin{aligned}
& \mathbb{L}_{(1,1)}:=\text { the set of even lattices of rank } 2 \text { and signature }(1,1), \\
& \mathbb{L}_{(1,1)}^{\star}:=\left\{L \in \mathbb{L}_{(1,1)} \mid v^{2} \notin\{0,-2\}(\forall v \in L)\right\} .
\end{aligned}
$$

Lemma 2.6. Let $K=\mathbb{Q}(\sqrt{D})$ be a real quadratic field of discriminant $D(>0)$. Then there exists $L \in \mathbb{L}_{(1,1)}^{\star}$ with $\operatorname{disc}(L)=-D$ if and only if $h^{+}(D)>1$. Here $h^{+}(D)$ denotes the narrow class number of $K$.

Proof. It is classically known that there is a natural bijection between the set of isomorphism classes of $L \in \mathbb{L}_{(1,1)}$ with $\operatorname{disc}(L)=-D$ and the narrow ideal class group of $K$ (see e.g. [FT, Section VII.2, Theorem 58]).

Let $L \in \mathbb{L}_{(1,1)}$. There exists $v \in L$ with $v^{2}=0$ if and only if $-\operatorname{disc}(L)$ is a square number. Hence, for any $L$ such that $\operatorname{disc}(L)=-D$ and $L \notin \mathbb{L}_{(1,1)}^{\star}$, there exists $v \in L$ with $v^{2}=-2$ and

$$
L \cong L_{0}:=\left(\begin{array}{cc}
-2 & \delta \\
\delta & \left(D-\delta^{2}\right) / 2
\end{array}\right)
$$

where $\delta \in\{0,1\}$ and $D \equiv \delta(\bmod 2)$. Therefore the existence of $L \in \mathbb{L}_{(1,1)}^{\star}$ with $\operatorname{disc}(L)=-D$ is equivalent to the existence of $L \in \mathbb{L}_{(1,1)}$ with $\operatorname{disc}(L)=-D$ and $L \nsubseteq L_{0}$, which is also equivalent to the condition $h^{+}(D)>1$.

\section{K3 SURFACES}

A compact complex surface $X$ is called a K3 surface if it is simply connected and has a nowhere vanishing holomorphic 2-from $\omega_{X}$ (see [BHPV] for details). We consider the second integral cohomology $H^{2}(X, \mathbb{Z})$ with the cup product as a lattice. It is known that $H^{2}(X, \mathbb{Z})$ is an even unimodular lattice of signature $(3,19)$, which is unique up to isomorphism and is called the K3 lattice. We fix such a lattice and denote it by $\Lambda_{\mathrm{K} 3}$. The Picard lattice $S_{X}$ and transcendental lattice $T_{X}$ of $X$ are defined by

$$
\begin{aligned}
& S_{X}:=\left\{x \in H^{2}(X, \mathbb{Z}) \mid\left\langle x, \omega_{X}\right\rangle=0\right\} \\
& T_{X}:=\left\{x \in H^{2}(X, \mathbb{Z}) \mid\langle x, y\rangle=0\left(\forall y \in S_{X}\right)\right\} .
\end{aligned}
$$


Here $\omega_{X}$ is considered as an element in $H^{2}(X, \mathbb{C})$ and the bilinear form on $H^{2}(X, \mathbb{Z})$ is extended to that on $H^{2}(X, \mathbb{C})$ linearly. The Picard group of $X$ is naturally isomorphic to $S_{X}$. It is known that $X$ is projective if and only if $S_{X}$ has signature $(1, \rho-1)$, where $\rho=\operatorname{rank} S_{X}$ is the Picard number of $X$.

Let $X$ be a projective $\mathrm{K} 3$ surface. Since $H^{2}(X, \mathbb{Z})$ is unimodular and $S_{X}$ is nondegenerate, we have the following natural identification:

$$
A\left(S_{X}\right)=A\left(T_{X}\right)=H^{2}(X, \mathbb{Z}) /\left(S_{X} \oplus T_{X}\right)
$$

(see [N2] for details). By the global Torelli theorem for K3 surfaces [PS, BR], the following map is injective:

$$
\operatorname{Aut}(X) \ni \varphi \mapsto(g, h):=\left(\left.\varphi^{*}\right|_{S_{X}},\left.\varphi^{*}\right|_{T_{X}}\right) \in O\left(S_{X}\right) \times O\left(T_{X}\right) .
$$

Moreover, $(g, h) \in O\left(S_{X}\right) \times O\left(T_{X}\right)$ is the image of some $\varphi \in \operatorname{Aut}(X)$ by the map (3.4) if and only if (1) the linear extension of $g$ (resp. $h$ ) preserves the ample cone $C_{X}$ of $X$ (resp. $\mathbb{C} \omega_{X}$ ) and (2) the actions of $g$ and $h$ on $A\left(S_{X}\right)=A\left(T_{X}\right)$ coincide.

In this paper, we study projective K3 surfaces $X$ with Picard number 2, for which we have $S_{X} \in \mathbb{L}_{(1,1)}$ (see (2.9) ). Conversely, the following holds:

Proposition 3.1. For any $L \in \mathbb{L}_{(1,1)}$, there exists a (projective) K3 surface $X$ such that $S_{X} \cong L$.

Proof. Let $L \in \mathbb{L}_{(1,1)}$. The K3 lattice $\Lambda_{\mathrm{K} 3}$ contains a primitive sublattice $S$ which is isomorphic to $L$ [N1, Theorem 1.14.4]. (In fact, such an $S$ is unique up to $O\left(\Lambda_{\mathrm{K} 3}\right)$.) The surjectivity of the period map for K3 surfaces [T] implies that there exists a K3 surface $X$ such that $S_{X} \cong L$.

Let $X$ be a projective K3 surface with Picard number 2. We are interested in automorphisms $\varphi \in \operatorname{Aut}(X)$ of infinite order such that $\varphi^{*} \omega_{X}=\varepsilon \omega_{X}$ with $\varepsilon \in\{ \pm 1\}$. By applying the global Torelli theorem, we obtain the following:

Proposition 3.2. Let $X$ be a projective K3 surface with Picard number 2 and $(g, h) \in$ $O\left(S_{X}\right) \times O\left(T_{X}\right)$. Then $(g, h)=\left(\left.\varphi^{*}\right|_{S_{X}},\left.\varphi^{*}\right|_{T_{X}}\right)$ for some $\varphi \in \operatorname{Aut}(X)$ such that $\operatorname{ord}(\varphi)=\infty$ and $\varphi^{*} \omega_{X}=\varepsilon \omega_{X}$ with $\varepsilon \in\{ \pm 1\}$ if and only if the following conditions are satisfied:

(1) $S_{X} \in \mathbb{L}_{(1,1)}^{\star}$;

(2) $1 \neq g \in S O^{+}\left(S_{X}\right)$;

(3) $g$ acts on $A\left(S_{X}\right)$ as $\varepsilon \cdot \mathrm{id}$;

(4) $h=\varepsilon \cdot \mathrm{id}$.

Proof. Assume that $(g, h)=\left(\left.\varphi^{*}\right|_{S_{X}},\left.\varphi^{*}\right|_{T_{X}}\right)$ for some $\varphi$ as in the statement. Since $\operatorname{Aut}(X)$ is infinite if and only if $S_{X} \in \mathbb{L}_{(1,1)}^{\star}$ [GLP, Corollary 3.4], the condition (1) holds. Moreover, the ample cone $C_{X}$ of $X$ coincides with $C_{S_{X}}$ or $-C_{S_{X}}$ (see (2.3)). Since $\varphi^{*} \omega_{X}=\varepsilon \omega_{X}, h$ acts on $T_{X}$ as $\varepsilon \cdot$ id [N1, Theorem 3.1]. Thus the condition (4) holds. This implies the condition (3) because the actions of $g$ and $h$ on $A\left(S_{X}\right)=A\left(T_{X}\right)$ coincide. If $\operatorname{det}(g)=-1$, then the action of $g$ on $C_{X}$ is a reflection, and hence $g^{2}=1$. Since $h^{2}=1$, we have $\varphi^{2}=1$ by the injectivity of the map (3.4), which is a contradiction. Therefore $g \in S O^{+}\left(S_{X}\right)$. Similarly, we have $g \neq 1$. Hence the condition (2) holds.

Conversely, if the conditions (1)-(4) are satisfied, then there exists $\varphi \in \operatorname{Aut}(X)$ such that $(g, h)=\left(\left.\varphi^{*}\right|_{S_{X}},\left.\varphi^{*}\right|_{T_{X}}\right)$ by the global Torelli theorem. The condition (4) 
implies that $\varphi^{*} \omega_{X}=\varepsilon \omega_{X}$ because $\omega_{X} \in T_{X} \otimes \mathbb{C}$. Since $S O^{+}\left(S_{X}\right)$ is isomorphic to $\mathbb{Z}$ (Proposition 2.2), $\varphi$ is of infinite order.

Remark 3.3. A non-projective K3 surface with Picard number 2 may have an automorphism of infinite order (cf. [M]).

\section{Proof of Main Theorem}

4.1. Preparation. In order to prove Main Theorem, we show the following:

Proposition 4.1. Let $X$ be a projective K3 surface with Picard number 2. For $\varepsilon \in\{ \pm 1\}$ and $g \in O\left(S_{X}\right)$, the following conditions are equivalent:

(1) $g=\left.\varphi^{*}\right|_{S_{X}}$ for some $\varphi \in \operatorname{Aut}(X)$ such that $\operatorname{ord}(\varphi)=\infty$ and $\varphi^{*} \omega_{X}=\varepsilon \omega_{X}$.

(2) $S_{X} \in \mathbb{L}_{(1,1)}^{\star}$ and $g$ is given by

$$
g=g_{u, v}:=\left(\begin{array}{cc}
(u-b v) / 2 & -c v \\
a v & (u+b v) / 2
\end{array}\right)
$$

where

$$
S_{X}=\left(\begin{array}{cc}
2 a & b \\
b & 2 c
\end{array}\right), \quad(u, v)=\left(\alpha^{2}-2 \varepsilon, \alpha \beta\right),
$$

and $\alpha, \beta$ are nonzero integers satisfying

$$
\alpha^{2}-D \beta^{2}=4 \varepsilon, \quad D:=-\operatorname{disc}\left(S_{X}\right)=b^{2}-4 a c>0 .
$$

Remark 4.2. Assume that $D$ is not a square number and $\operatorname{gcd}(a, b, c)=1$ in Proposition 4.1, Then Proposition 2.2 implies that

$$
S O^{+}\left(S_{X}\right)=\left\{g_{u, v} \mid u, v \in \mathbb{Z}, u>0, u^{2}-D v^{2}=4\right\} \cong \mathbb{Z} .
$$

Under the condition (2) in Proposition 4.1, we have

$$
\left(\frac{\alpha+\beta \sqrt{D}}{2}\right)^{2}=\frac{\alpha^{2}-2 \varepsilon+\alpha \beta \sqrt{D}}{2}=\frac{u+v \sqrt{D}}{2} .
$$

Hence $g_{u, v}=g_{\alpha, \beta}^{2}$ (see Remark 2.5) , and thus

$$
\begin{aligned}
\Gamma & :=\left\{g \in S O^{+}\left(S_{X}\right)\left|g=\varphi^{*}\right|_{S_{X}}, \varphi^{*} \omega_{X}= \pm \omega_{X}(\exists \varphi \in \operatorname{Aut}(X))\right\} \\
& =\left\{g_{\alpha, \beta}^{2} \mid \alpha, \beta \in \mathbb{Z}, \alpha^{2}-D \beta^{2}= \pm 4\right\} .
\end{aligned}
$$

Therefore $\Gamma$ is a subgroup of $S O^{+}\left(S_{X}\right)$ of index 1 or 2 according to whether the equation $\alpha^{2}-D \beta^{2}=-4$ has an integer solution $(\alpha, \beta)$ or not.

We obtain Proposition 4.3 below from Proposition 4.1, because any lattice $L \in$ $\mathbb{L}_{(1,1)}$ is realized as the Picard lattice $S_{X}$ of a $\mathrm{K} 3$ surface $X$ by Proposition 3.1 .

Proposition 4.3. For $\varepsilon \in\{ \pm 1\}$ and $u \in \mathbb{Z}$, the following conditions are equivalent:

(1) $u=\operatorname{trace}\left(\left.\varphi^{*}\right|_{S_{X}}\right)$ for some automorphism $\varphi$ of a projective K3 surface $X$ with Picard number 2 such that $\operatorname{ord}(\varphi)=\infty$ and $\varphi^{*} \omega_{X}=\varepsilon \omega_{X}$.

(2) $u=\alpha^{2}-2 \varepsilon$ for some $\alpha \in \mathbb{Z}_{>0}$ such that there exist $L \in \mathbb{L}_{(1,1)}^{\star}$ and $\beta \in \mathbb{Z}_{>0}$ satisfying $\operatorname{disc}(L)=-\left(\alpha^{2}-4 \varepsilon\right) / \beta^{2}$.

Proposition 4.1 is a direct conclusion of Proposition 3.2 and the following: 
Lemma 4.4. Let $L \in \mathbb{L}_{(1,1)}$ :

$$
L=\left(\begin{array}{cc}
2 a & b \\
b & 2 c
\end{array}\right), \quad D:=-\operatorname{disc}(L)=b^{2}-4 a c>0,
$$

and let $g$ be a nontrivial isometry contained in $\mathrm{SO}^{+}(L)$ (see Proposition 2.2):

$$
g=\left(\begin{array}{cc}
(u-b v) / 2 & -c v \\
a v & (u+b v) / 2
\end{array}\right), \quad u^{2}-D v^{2}=4, \quad u>2 .
$$

For $\varepsilon \in\{ \pm 1\}$, the isometry $g$ acts on $A(L)$ as $\varepsilon \cdot$ id if and only if we have

$$
(u, v)=\left(\alpha^{2}-2 \varepsilon, \alpha \beta\right), \quad \alpha^{2}-D \beta^{2}=4 \varepsilon
$$

for some nonzero integers $\alpha, \beta$.

Proof. Recall that we have $u, k v \in \mathbb{Z}$ and $u>0$, where $k:=\operatorname{gcd}(a, b, c)$. Moreover, since $g \neq 1$, it follows that $u>2$. We have

$$
\operatorname{det}\left(g-\varepsilon \cdot I_{2}\right)=\operatorname{det}(g)-\varepsilon \cdot \operatorname{trace}(g)+1=-\varepsilon u+2
$$

and

$$
\begin{aligned}
\left(g-\varepsilon \cdot I_{2}\right) \cdot Q_{L}^{-1} & =\frac{-1}{D}\left(\begin{array}{cc}
(u-b v) / 2-\varepsilon & -c v \\
a v & (u+b v) / 2-\varepsilon
\end{array}\right)\left(\begin{array}{cc}
2 c & -b \\
-b & 2 a
\end{array}\right) \\
& =\frac{-1}{D}\left(\begin{array}{cc}
c(u-2 \varepsilon) & (D v-b(u-2 \varepsilon)) / 2 \\
-(D v+b(u-2 \varepsilon)) / 2 & a(u-2 \varepsilon)
\end{array}\right) .
\end{aligned}
$$

Assume that $g$ acts on $A(L)$ as $\varepsilon \cdot$ id. By Lemma 2.1, $\left(g-\varepsilon \cdot I_{2}\right) \cdot Q_{L}^{-1}$ is an integer matrix, and hence $D$ divides $\operatorname{det}\left(g-\varepsilon \cdot I_{2}\right)=-\varepsilon u+2$. Thus $u-2 \varepsilon=m D$ for some $m \in \mathbb{Z}_{>0}$. Therefore

$$
v^{2}=\frac{1}{D}\left(u^{2}-4\right)=m(m D+4 \varepsilon) .
$$

In particular, we have $v \in \mathbb{Z}$.

Claim. $m$ is a square number.

This is shown by applying (4.14) as follows. Suppose that $p$ is a prime number and $p^{e} \| m$ with $e \in \mathbb{Z}_{>0}$ (that is, $p^{e} \mid m$ and $p \nmid\left(m / p^{e}\right)$ ). It is enough to show that $e$ is always even. (i) If $p$ is odd, then $p^{e} \| m(m D+4 \varepsilon)=v^{2}$. Hence $e$ is even. (ii) In the case $p=2$, if $e \geq 3$, then $m D+4 \varepsilon \equiv 4(\bmod 8)$. Hence $2^{e+2} \| m(m D+4 \varepsilon)=v^{2}$, and thus $e$ is even. If $e=1$, then $(v / 2)^{2}=(m / 2)^{2} D+\varepsilon m \equiv 2$ or $3(\bmod 4)$ because $D=b^{2}-4 a c \equiv 0$ or $1(\bmod 4)$. On the other hand, $v$ is even and $(v / 2)^{2} \equiv 0$ or 1 $(\bmod 4)$, which is a contradiction. This completes the proof of Claim.

By Claim, we have $m=n^{2}$ for some $n \in \mathbb{Z}_{>0}$ and

$$
u-2 \varepsilon=n^{2} D \text {. }
$$

By (4.14), we have $v^{2}=n^{2}\left(n^{2} D+4 \varepsilon\right)$. Thus $v=\alpha \beta$ and $n^{2} D+4 \varepsilon=\alpha^{2}$ for some $\alpha \in \mathbb{Z}_{>0}$ and $\beta \in\{ \pm n\}$. This implies (4.10).

Conversely, assume that $D$ and $(u, v)$ satisfy (4.10) for some nonzero integers $\alpha, \beta$. In order to show that $g$ acts on $A(L)$ as $\varepsilon \cdot \mathrm{id}$, it is enough to check that the matrix (4.13) is an integer matrix by Lemma 2.1. By (4.10), we have

$$
\frac{u-2 \varepsilon}{D}=\beta^{2}, \quad \frac{D v \pm b(u-2 \varepsilon)}{2 D}=\frac{v \pm b \beta^{2}}{2} .
$$


If $v \pm b \beta^{2}$ is odd, then

$$
1 \equiv v \pm b \beta^{2} \equiv \alpha \beta+b \beta=(\alpha+b) \beta, \quad \alpha+b \equiv \beta \equiv 1 \quad(\bmod 2),
$$

and thus

$$
\alpha+1 \equiv b \equiv b^{2}-4 a c=D=\left(\alpha^{2}-4 \varepsilon\right) / \beta^{2} \equiv \alpha^{2}-4 \varepsilon \equiv \alpha \quad(\bmod 2),
$$

which is a contradiction. Hence $v \pm b \beta^{2}$ is even and the matrix (4.13) is an integer matrix. Therefore $g$ acts on $A(L)$ as $\varepsilon \cdot$ id.

Thanks to Proposition 4.3, the proof of Main Theorem is reduced to showing

$$
\begin{aligned}
A_{\varepsilon} & :=\left\{\alpha \in \mathbb{Z}_{>0} \mid \operatorname{disc}(L)=-\left(\alpha^{2}-4 \varepsilon\right) / \beta^{2}\left(\exists \beta \in \mathbb{Z}_{>0}, \exists L \in \mathbb{L}_{(1,1)}^{\star}\right)\right\} \\
& = \begin{cases}\mathbb{Z}_{\geq 4} & \text { if } \varepsilon=1, \\
\mathbb{Z}_{\geq 4} \backslash\{5,7,13,17\} & \text { if } \varepsilon=-1 .\end{cases}
\end{aligned}
$$

4.2. Symplectic case. For $\alpha \in A_{+1}$, we have $\alpha \geq 3$. If $\alpha=3$, then there exists $L \in \mathbb{L}_{(1,1)}^{\star}$ such that $\operatorname{disc}(L)=-5$. However, it follows from the table of indefinite binary quadratic forms [CS, Table 15.2] that

$$
L \cong\left(\begin{array}{cc}
2 & 1 \\
1 & -2
\end{array}\right) \notin \mathbb{L}_{(1,1)}^{\star}
$$

which is a contradiction. Hence $\alpha \geq 4$.

Conversely, for $\alpha \in \mathbb{Z}_{\geq 4}$, we set

$$
L:=\left(\begin{array}{cc}
2 & \alpha \\
\alpha & 2
\end{array}\right) \in \mathbb{L}_{(1,1)}, \quad \operatorname{disc}(L)=-\left(\alpha^{2}-4\right) .
$$

By [GLP, Example 4], we have $L \in \mathbb{L}_{(1,1)}^{\star}$, and thus $\alpha \in A_{+1}$.

4.3. Anti-symplectic case. Now we consider the case $\varepsilon=-1$. Let $\alpha \in \mathbb{Z}_{>0}$.

4.3.1. Case of odd $\alpha$. Suppose that $\alpha$ is odd.

First we assume that $\alpha^{2}+4$ is not square-free, that is, $D:=\alpha^{2}+4=n^{2} D_{0}$ with $n>1$. Since $D \equiv 1(\bmod 4)$, we have $D_{0} \equiv 1(\bmod 4)$. We define an even lattice $L$ by

$$
L:=\left(\begin{array}{cc}
2 n & n \\
n & -n\left(D_{0}-1\right) / 2
\end{array}\right), \quad \operatorname{disc}(L)=-n^{2} D_{0}=-D .
$$

Since $D$ is not a square number, it follows that $L \in \mathbb{L}_{(1,1)}^{\star}$. Hence $\alpha \in A_{-1}$ in this case.

Next we assume that $D:=\alpha^{2}+4$ is square-free. Then $D$ is the discriminant of the real quadratic field $\mathbb{Q}(\sqrt{D})$. By Lemma 2.6, there exists $L \in \mathbb{L}_{(1,1)}^{\star}$ with $\operatorname{disc}(L)=-D$ if and only if $h^{+}(D)>1$. Therefore, in this case, the conditions $\alpha \in A_{-1}$ and $\alpha \notin\{1,3,5,7,13,17\}$ are equivalent by Theorem 4.5 (and Remark 4.6) below.

Theorem 4.5. ([Bi] For an odd integer $k>0$ such that $D:=k^{2}+4$ is square-free, the class number $h(D)$ of $\mathbb{Q}(\sqrt{D})$ is 1 if and only if $k \in\{1,3,5,7,13,17\}$.

Remark 4.6. In Theorem 4.5, since the equation $u^{2}-D v^{2}=-4$ has an integer solution (e.g. $(u, v)=(k, 1))$, we have $h^{+}(D)=h(D)$. 
4.3.2. Case of even $\alpha$. Suppose that $\alpha$ is even and $\alpha \in A_{-1}$. If $\alpha=2$, then there exists $L \in \mathbb{L}_{(1,1)}^{\star}$ such that $\operatorname{disc}(L)=-8$ or -2 . By [CS, Table 15.2], we have

$$
L \cong\left(\begin{array}{cc}
-2 & 0 \\
0 & 4
\end{array}\right) \notin \mathbb{L}_{(1,1)}^{\star}
$$

which is a contradiction. Therefore $\alpha \geq 4$.

Conversely, for even $\alpha \geq 4$, we define an even lattice $L$ by

$$
L:=\left(\begin{array}{cc}
\alpha & 2 \\
2 & -\alpha
\end{array}\right), \quad \operatorname{disc}(L)=-\left(\alpha^{2}+4\right)
$$

We show $L \in \mathbb{L}_{(1,1)}^{\star}$. It is easy to check this for the case that $\alpha$ is divisible by 4 . In the case $\alpha \equiv 2(\bmod 4)$, we consider the following Gram matrix of $L$ :

$$
P^{T} \cdot\left(\begin{array}{cc}
\alpha & 2 \\
2 & -\alpha
\end{array}\right) \cdot P=\left(\begin{array}{cc}
4 & 0 \\
0 & -\left(\alpha^{2} / 4+1\right)
\end{array}\right), \quad \text { where } P=\left(\begin{array}{cc}
1 & (\alpha-2) / 4 \\
1 & (\alpha+2) / 4
\end{array}\right)
$$

We can apply Theorem 4.7 below for $\Delta=\alpha^{2} / 4+1, a=2$ and $b=\left(\alpha^{2} / 4+1\right) / 2$ to show $L \in \mathbb{L}_{(1,1)}^{\star}$. Indeed, we have the continued fraction expansion

$$
\sqrt{\alpha^{2} / 4+1}=[\alpha / 2, \alpha, \alpha, \alpha, \ldots] .
$$

The length of the period of this continued fraction is 1 .

Theorem 4.7. ([Mo]) Suppose that an integer $\Delta>2$ is not a square number. Then the length of the period of the continued fraction expansion of $\sqrt{\Delta}$ is even if and only if one of the following holds.

(1) There exists a factorization $\Delta=a b$ with $1<a<b$ such that

$$
a x^{2}-b y^{2}= \pm 1
$$

has an integer solution.

(2) There exists a factorization $\Delta=a b$ with $1 \leq a<b$ suth that

$$
a x^{2}-b y^{2}= \pm 2
$$

has an integer solution with $x y$ odd.

\section{Applications}

In this section we discuss applications of our result.

First we give direct consequences of Main Theorem. Let $\varphi$ be as in Main Theorem, that is, $\varphi$ is an automorphism of a projective K3 surface $X$ with Picard number 2 such that $\operatorname{ord}(\varphi)=\infty$ and $\varphi^{*} \omega_{X}=\varepsilon \omega_{X}(\varepsilon \in\{ \pm 1\})$. Then $\operatorname{trace}\left(\left.\varphi^{*}\right|_{S_{X}}\right)=\alpha^{2}-2 \varepsilon$ for some $\alpha \in A_{\varepsilon}$. By [0], each fixed point of $\varphi$ on $X$ is isolated. By the topological Lefschetz fixed point formula, the number of fixed points (with multiplicity) of $\varphi$ is given as

$$
\sum_{i=0}^{4}(-1)^{i} \operatorname{trace}\left(\left.\varphi^{*}\right|_{H^{i}(X, \mathbb{C})}\right)=1+\left(20 \varepsilon+\operatorname{trace}\left(\left.\varphi^{*}\right|_{S_{X}}\right)\right)+1=\alpha^{2}+18 \varepsilon+2 .
$$

As another consequence, we determine the spectral radius of $\left.\varphi^{*}\right|_{H^{2}(X, \mathbb{C})}$, which is defined as the maximum absolute value $|\lambda|$ of eigenvalues $\lambda$ of $\left.\varphi^{*}\right|_{H^{2}(X, \mathbb{C})}$. This plays 
an important role in the study of complex dynamics of K3 surfaces (see [M]). In our case, the spectral radius is given as

$$
\frac{u+\sqrt{u^{2}-4}}{2}=\frac{\alpha^{2}-2 \varepsilon+\alpha \sqrt{\alpha^{2}-4 \varepsilon}}{2}
$$

where $u=\alpha^{2}-2 \varepsilon$.

Next we show that a fixed-point-free automorphism of a projective K3 surface with Picard number 2 is nothing but the following Cayley-Oguiso automorphism:

Theorem 5.1. ([0]) Any K3 surface $X$ with

$$
S_{X} \cong\left(\begin{array}{cc}
4 & 2 \\
2 & -4
\end{array}\right)
$$

admits a fixed-point-free automorphism $\varphi$ of positive entropy with

$$
\left.\varphi^{*}\right|_{S_{X}}=\left(\begin{array}{cc}
5 & 8 \\
8 & 13
\end{array}\right),\left.\quad \varphi^{*}\right|_{T_{X}}=-\mathrm{id} .
$$

Remark 5.2. In Theorem 5.1, Aut $(X)$ is generated by $\varphi$ [FGvGvL, Theorem 1.1].

Theorem 5.3. Any fixed-point-free automorphism $\varphi$ of a projective K3 surface $X$ with Picard number 2 is of the form in Theorem 5.1.

Proof. By [0], we have trace $\left(\left.\varphi^{*}\right|_{S_{X}}\right)=18$ and $\varphi^{*} \omega_{X}=-\omega_{X}$. Hence $\operatorname{disc}\left(S_{X}\right)=-20$ or -5 by Proposition 4.1. From [CS, Table 15.2], we find that a lattice belonging to $\mathbb{L}_{(1,1)}$ with discriminant -20 or -5 is isomorphic to one of the following:

$$
\left(\begin{array}{cc}
4 & 2 \\
2 & -4
\end{array}\right),\left(\begin{array}{cc}
-2 & 0 \\
0 & 10
\end{array}\right),\left(\begin{array}{cc}
2 & 1 \\
1 & -2
\end{array}\right) \text {. }
$$

Since $S_{X} \in \mathbb{L}_{(1,1)}^{\star}$ by Proposition 3.2 , we have

$$
S_{X} \cong\left(\begin{array}{cc}
4 & 2 \\
2 & -4
\end{array}\right),\left.\quad \varphi^{*}\right|_{S_{X}}=U \text { or } U^{-1}, \quad U:=\left(\begin{array}{cc}
5 & 8 \\
8 & 13
\end{array}\right)
$$

by Proposition 4.1. Here $U$ and $U^{-1}$ correspond to $(u, v)=(18, \pm 4)$ satisfying $u^{2}-20 v^{2}=4$. Since $O^{+}\left(S_{X}\right)$ is isomorphic to the infinite dihedral group, $U$ and $U^{-1}$ are conjugate in $O^{+}\left(S_{X}\right)$. In fact, we have

$$
V:=\left(\begin{array}{cc}
1 & 0 \\
1 & -1
\end{array}\right) \in O^{+}\left(S_{X}\right), \quad V^{-1} \cdot U^{-1} \cdot V=U .
$$

Hence we may assume $\left.\varphi^{*}\right|_{S_{X}}=U$ by changing basis of $S_{X}$ if necessary. We have $\left.\varphi^{*}\right|_{T_{X}}=-$ id by Proposition 3.2.

Remark 5.4. One can show that the action of a Cayley-Oguiso automorphism (as in Theorem 5.1) on the K3 lattice is essentially unique by applying Nikulin's lattice theory [N2]. Combined with Theorem [5.3, this implies that a pair $(X, \varphi)$ of a $\mathrm{K} 3$ surface $X$ with Picard number 2 and a fixed-point-free automorphism $\varphi \in \operatorname{Aut}(X)$ is unique up to equivariant deformation. Moreover, $X$ is realized as a determinantal quartic surface and $\varphi$ is constructed by using cofactor matrix. See [FGvGvL] for details. 


\section{REFERENCES}

[BHPV] W. P. Barth, K. Hulek, C. A. M. Peters and A. van de Ven, Compact complex surfaces, Second edition, Springer-Verlag, Berlin, 2004.

[Bi] A. Biro, Yokoi's conjecture, Acta Arithmetica, 106.1 (2003), 85-104.

[BR] D. Burns and M. Rapoport, On the Torelli problem for kählerian K-3 surfaces, Ann. Sci. Ecole Norm. Sup. (4) 8 (1975), no. 2, 235-273.

[CS] J. H. Conway and N. J. Sloane, Sphere packings, lattices and groups, Third edition, Springer-Verlag, New York, 1999.

[D] L. E. Dickson, Introduction to the theory of numbers, Dover, 1954.

[FGvGvL] D. Festi, A. Garbagnati, B. van Geemen and R. van Luijk, The Cayley-Oguiso automorphism of positive entropy on a K3 surface, J. Mod. Dyn. 7 (2013), no. 1, 75-97.

[FT] A. Fröhlich and M. J. Taylor, Algebraic number theory, Cambridge University Press, 1993.

[GLP] F. Galluzzi, G. Lombardo, C. Peters, Automorphs of indefinite binary quadratic forms and K3-surfaces with Picard number 2, Rend. Sem. Mat. Univ. Politec. Torino, 68(1) (2010), 57-77.

[J] B. W. Jones, The arithmetic theory of quadratic forms. Carcus Monograph Series, no. 10. The mathematical Association of America, Buffalo, N.Y., 1950.

[M] C. T. McMullen, Dynamics on K3 surfaces: Salem numbers and Siegel disks, J. Reine Angew. Math., 545 (2002), 201-233.

[Mo] R. A. Mollin, A continued fraction approach to the Diophantine equation $a x^{2}-b y^{2}= \pm 1$, JP Journal of Algebra, Number Theory and Applications, 4 (2004), 159-207.

[N1] V. V. Nikuiln, Finite groups of Kählerian surfaces of type K3 (English translation), Trans. Moscow Math. Soc. 38 (1980), 71-137.

[N2] V. V. Nikulin, Integral Symmetric bilinear forms and some of their applications (English translation), Math. USSR Izv. 14 (1980), 103-167.

[O] K. Oguiso, Free Automorphisms of positive entropy on smooth Kähler surfaces, arXiv:1202.2637, to appear in Adv. Stud. Pure Math.

[PS] I. I. Pjateckii-Šapiro and I. R. Šhafarevič, Torelli's theorem for algebraic surfaces of type K3 (in Russian), Izv. Akad. Nauk SSSR Ser. Mat. 35 (1971), 530-572.

[T] A. Todorov, Applications of the Kähler-Einstein-Calabi-Yau metric to moduli of K3 surfaces, Invent. Math., 61 (1980), 251-265.

Kenji Hashimoto

Graduate School of Mathematical Sciences, The University of Tokyo,

3-8-1 Komaba, Maguro-ku, Tokyo, 153-8914, Japan

hashi@ms.u-tokyo.ac.jp

JongHae Keum

School of Mathematics,

Korea Institute for Advanced Study,

85 Hoegiro, Dongdaemun-gu, Seoul 02455, Korea

jhkeum@kias.re.kr

Kwangwoo Lee

Division of Liberal Arts and Science, Gwangju Institute of Science and Technology, 123 Cheomdangwagi-ro, Buk-gu, Gwangju 61005, Korea kwlee@gist.ac.kr 